Article

\title{
Quality Evaluation of Tricholoma matsutake Based on the Nucleic Acid Compounds by UPLC-TOF/MS and UPLC-QqQ/MS
}

\author{
Ying Xue ${ }^{1,2,3}$, Wei Jin ${ }^{3}$, Xian-Shun $\mathrm{Xu}^{3}{ }^{3}$ Li Yong $^{3}{ }^{3}$ Bin $\mathrm{Hu}^{3}{ }^{3}$ Jing Xiong ${ }^{1}$, Xue-Mei Hu ${ }^{1}$, \\ Lin-Sen Qing ${ }^{1,2, *}$ and Jing Xie ${ }^{1, *}$ \\ 1 School of Pharmacy, Collaborative Innovation Center of Sichuan for Elderly Care and Health, \\ Chengdu Medical College, Chengdu 610500, China; xuecher0221@sina.com (Y.X.); \\ s_scu_xiongjing@163.com (J.X.); xuemei.hu@163.com (X.-M.H.) \\ 2 Chengdu Institute of Biology, Chinese Academy of Sciences, Chengdu 610041, China \\ 3 Sichuan Provincial Center for Disease Control and Prevention, Chengdu 610041, China; \\ qgmw6970@163.com (W.J.); xuxianshun@163.com (X.-S.X.); yongch121@163.com (L.Y.); \\ hubin1258@163.com (B.H.) \\ * Correspondence: qingls@cib.ac.cn (L.-S.Q.); aggie-xj@163.com (J.X.); \\ Tel.: +86-28-82890640 (L.-S.Q.); +86-28-62308658 (J.X.)
}

Academic Editor: Chi-Fai Chau

Received: 8 December 2018; Accepted: 20 December 2018; Published: 21 December 2018

\begin{abstract}
So far, there has been no quality evaluation of Tricholoma matsutake. Nucleic acid compounds are a kind of functional ingredient in T. matsutake that is beneficial to human health. In this study, a UPLC-TOF/MS method was first used to scan and identify the potential nucleic acid compounds in T. matsutake. Based on the calculation of the molecular formula and subsequent confirmation by authentic standards, 15 nucleic acid compounds were unambiguously identified: adenosine, cytidine, guanosine, inosine, thymidine, uridine, xanthosine dehydrate, $2^{\prime}$-deoxyadenosine, $2^{\prime}$-deoxycytidine, $2^{\prime}$-deoxyguanosine, $2^{\prime}$-deoxyuridine, adenosine $5^{\prime}$-monophosphate, cytidine $5^{\prime}$-monophosphate, guanosine $5^{\prime}$-monophosphate, and uridine $5^{\prime}$-monophosphate. Then, a UPLC-QqQ/MS method was developed for the subsequent quantitative analysis. After validating the limits of quantification, detection, precision, repeatability, and recovery through a calibration curve, the content of 15 nucleic acid compounds was determined by the proposed UPLC-QqQ/MS method in 80 T. matsutake samples collected from different regions in Sichuan province, Southwest China. After the statistical analysis, we suggest that the total content of nucleic acid compounds in the qualified T. matsutake should be higher than $24.49 \mathrm{mg} / 100 \mathrm{~g}$. The results indicated that the combined use of UPLC-TOF/MS and UPLC-QqQ/MS is efficient for fast identification and determination of nucleic acid compounds to comprehensively evaluate the quality of T. matsutake.
\end{abstract}

Keywords: Tricholoma matsutake; quality evaluation; nucleic acid compound; UPLC-TOF/MS; UPLC-QqQ/MS

\section{Introduction}

Tricholoma matsutake, a natural, wild, edible fungus, is an endemic species in East Asia. The Hengduan Mountain range region of Southwest China, especially the Sichuan province, is the world's main production area of T. matsutake. China, Europe, and Japan are the world's three major consumer markets. Tricholoma matsutake is widely used in high-end food because of its unique flavor and fresh taste. Additionally, it is also made into health products with diverse biological activities, such as antioxidant $[1,2]$, immuno-modulating [3,4], and anti-tumor $[5,6]$ activity. However, so far, there has been no quality evaluation of $T$. matsutake. 
Ying et al. reported 5 nucleic acid constituents of T. matsutake for the first and only time [7]. Nucleic acid components are the most important functional small molecule ingredients in edible fungi. Nucleic acid components are made of DNA and RNA precursor molecules and play crucial roles in almost all cellular functions. They regulate various physiological processes through the purine/pyrimidine receptors in the human body [8,9]. Eating foods rich in nucleic acid compounds can help in the recovery from diseases and enhance the immune system. Therefore, nucleic acid constituents are suitable and quantifiable markers for the evaluation of the quality of T. matsutake.

Sichuan Food and Drug Administration commissioned our team and other researchers to jointly draft the local standards for the safety of T. matsutake and its products, which were released and implemented on 20 July 2018 [10]. These local standards only stipulate food safety requirements such as pesticide residues, heavy metal residues, and microbial limits, etc. Time-of-flight mass spectrometry is commonly used in the discovery of unknown compounds in a complex matrix [11]. When target compounds are located and identified, QqQ/MS is an excellent choice for the subsequent quantitative analysis [12]. In this study, 15 nucleic acid compounds in 80 T. matsutake samples from different regions from Sichuan province were firstly screened by UPLC-TOF/MS and subsequently determined by UPLC-QqQ/MS to comprehensively evaluate the quality of T. matsutake.

\section{Results and Discussion}

\subsection{Scanning and Identification of Nucleic Acid Compounds by UPLC-TOF/MS}

Since only one research article has preliminarily reported the existence of 5 nucleic acid compounds in T. matsutake [7], UPLC-TOF/MS was first used to scan and identify the potential nucleic acid compounds in T. matsutake. TOF/MS is often used for qualitative analysis, since it is able to determine the high-resolution molecular mass of target compounds, enabling qualitative structure identification in a complex matrix [13]. When target compounds are located and identified, QqQ/MS is an excellent choice for the subsequent quantitative analysis [14].

In this study, based on the calculation of the molecular formula and subsequent confirmation by authentic standards under the same chromatographic conditions, 15 nucleic acid compounds (Figure 1) were unambiguously identified in T. matsutake: adenosine (A), cytidine (C), guanosine $(G)$, inosine $(\mathrm{I})$, thymidine $(\mathrm{T})$, uridine $(\mathrm{U})$, xanthosine dehydrate $(\mathrm{X}), 2^{\prime}$-deoxyadenosine $(\mathrm{dA}), 2^{\prime}$-deoxycytidine (dC), 2'-deoxyguanosine (dG), 2'-deoxyuridine (dU), adenosine $5^{\prime}$-monophosphate (AMP), cytidine $5^{\prime}$-monophosphate (CMP), guanosine 5'-monophosphate (GMP), and uridine $5^{\prime}$-monophosphate (UMP). As shown in Table 1, the error of each compound in high-resolution mass spectral data was within $\pm 5 \mathrm{ppm}$, which is an acceptable error limit for structure confirmation $[15,16]$.

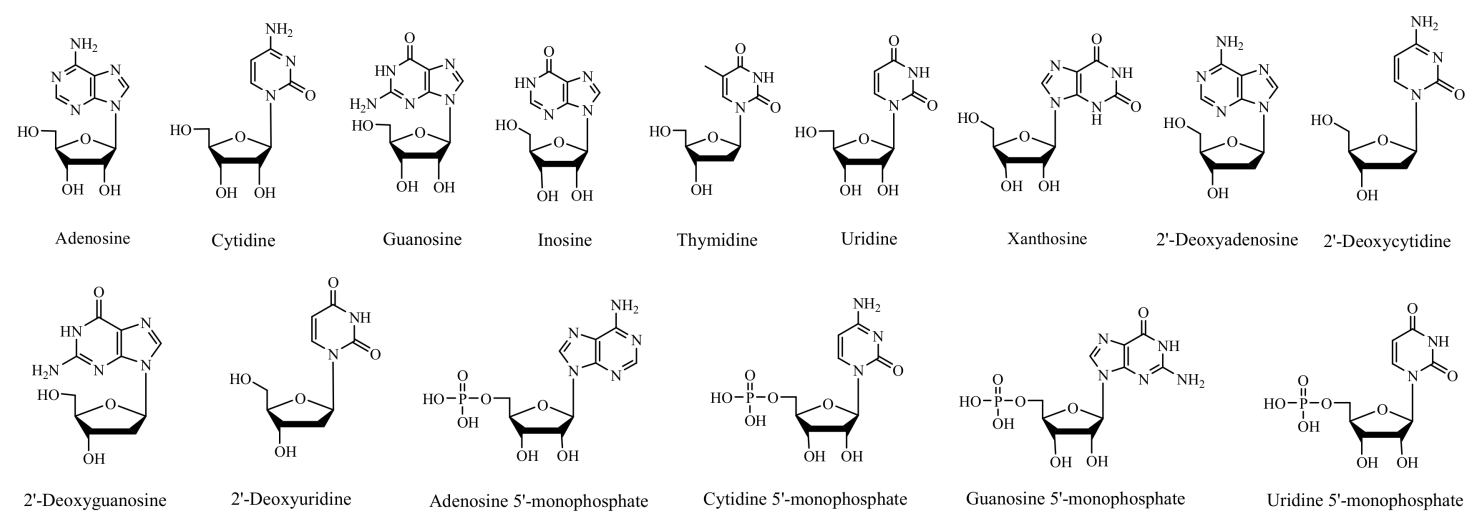

Figure 1. Chemical structures of 15 nucleic acid constituents. 
Table 1. Mass spectrometric parameters of 15 nucleic acid constituents in the negative ion-scan mode.

\begin{tabular}{ccccc}
\hline \multirow{2}{*}{ Analyte } & \multicolumn{2}{c}{ TOF/MS } & \multicolumn{2}{c}{ QqQ/MS } \\
\cline { 2 - 5 } & Quasi-Molecular Ion $(\mathbf{m} / \mathbf{z})$ & Error $(\mathbf{p p m})$ & Parent Ion $(\mathbf{m} / \mathbf{z})$ & Product Ion $(\mathbf{m} / \mathbf{z})$ \\
\hline A & 266.09019 & 2.7 & 266 & $134^{*}, 107^{\#}$ \\
C & 242.07717 & -4.4 & 242 & $152^{\#}, 109^{*}$ \\
G & 282.08436 & -0.1 & 282 & $133^{\#}, 150^{*}$ \\
I & 267.07231 & -4.4 & 267 & $135^{*}, 92^{\#}$ \\
T & 241.08235 & -2.7 & 241 & $151^{*}, 125^{\#}$ \\
U & 243.06279 & 2.2 & 243 & $152^{\#}, 110^{*}$ \\
X & 283.06741 & -3.5 & 283 & $151^{*}, 108^{\#}$ \\
dA & 250.09446 & -0.4 & 250 & $160^{\#}, 134^{*}$ \\
dC & 226.08297 & -1.6 & 226 & $135^{\#}, 93^{*}$ \\
dG & 266.08899 & -1.8 & 266 & $150^{*}, 133^{\#}$ \\
dU & 227.06625 & -4.8 & 227 & $184^{*}, 94^{\#}$ \\
AMP & 346.05423 & -4.6 & 346 & $211^{\#}, 79^{*}$ \\
CMP & 322.04404 & -1.7 & 322 & $211^{\#}, 79^{*}$ \\
GMP & 362.0499 & -2.3 & 362 & $211^{\#}, 79^{*}$ \\
UMP & 323.02825 & -1.1 & 323 & $211^{\#}, 79^{*}$ \\
\hline
\end{tabular}

Note: * quantitative ion, ${ }^{\#}$ qualitative ion.

\subsection{Determination of Nucleic Acid Compounds by UPLC-QqQ/MS}

\subsubsection{Optimization of the UPLC-QqQ/MS Condition}

After optimizing the types of analytical column, the solvent composition, and the $\mathrm{pH}$ of the mobile phase and the MS parameters, the UPLC-QqQ/MS conditions for the quantitative analysis of nucleic acid compounds were determined [14,17]. The typical mass spectra are shown in Figure 2.
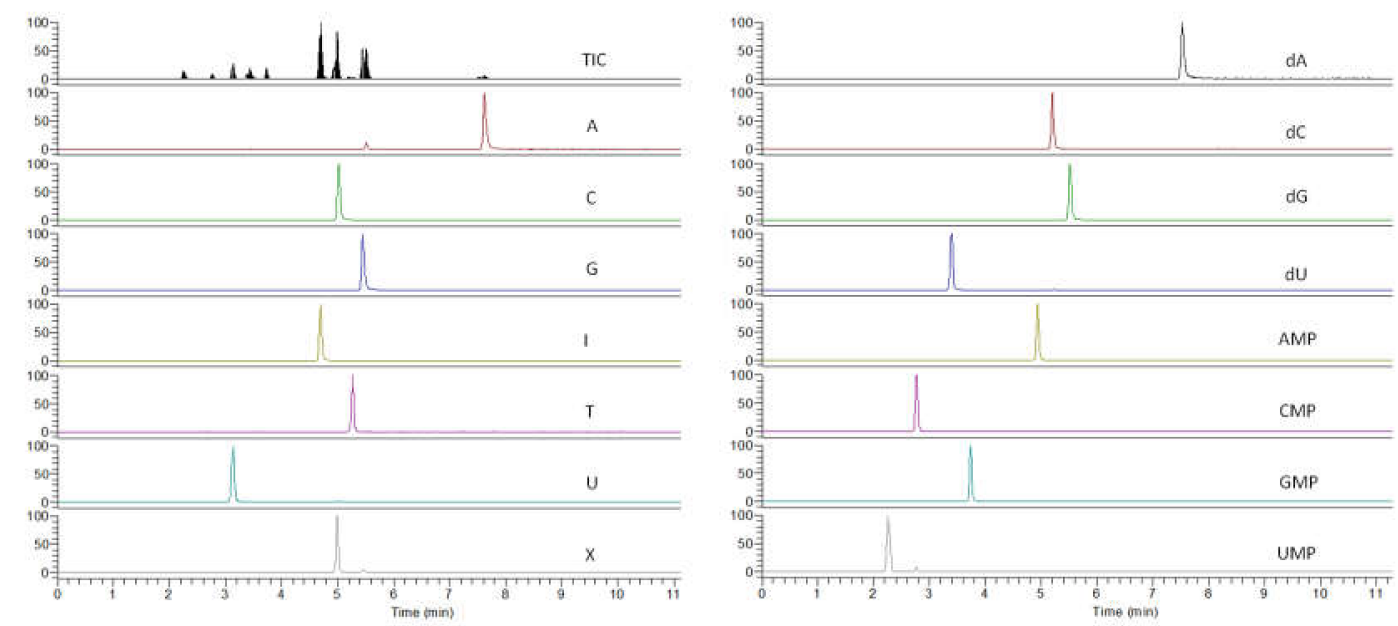

Figure 2. Ultra-performance liquid chromatography mass spectrometer chromatograms of 15 nucleic acid constituents.

\subsubsection{Analytical Figures of Merit}

Method validation was implemented under the above UPLC-QqQ/MS conditions. The calibration curves of 15 target analytes were firstly determined by the mixed working solutions I-V. Binary linear regression analysis was performed as shown in Table 2 by taking the concentration of each authentic standard as the abscissa $(x)$ and the corresponding peak area as the ordinate $(y)$, respectively. The limit of quantification (LOQ) and the limit of detection (LOD) were measured by a gradual dilution process of the standard stock solutions until the signal-to-noise ratio $(\mathrm{S} / \mathrm{N})$ was 10:1 and 3:1, respectively. The precision was evaluated by the relative standard deviation (RSD) using standard working solution 
III, which was tested within one day $(0 \mathrm{~h}, 4 \mathrm{~h}, 8 \mathrm{~h}, 12 \mathrm{~h}, 16 \mathrm{~h}$, and $24 \mathrm{~h})$ to determine the intra-day precision, and was tested within 3 days to determine the inter-day precision. The repeatability was evaluated by analyzing six independent portions of sample S- 05 . The recovery was evaluated by spiking an amount of about 1:1 of authentic standards to sample S-05 in parallel operation six times. The results summarized in Table 2 show that the analytical method meets the requirements for a quantitative analysis and was appropriate for the determination of 15 nucleic acid constituents in T. matsutake. Besides, the key values of method validation in this work were compared with those of several other quantitative methods reported, as shown in Table S1. 
Table 2. The results of method validation.

\begin{tabular}{|c|c|c|c|c|c|c|c|c|c|c|}
\hline \multirow{2}{*}{ Analyte } & \multirow{2}{*}{$\begin{array}{c}\text { Regression Equation } \\
\left(y=a x+b, r^{2}\right)\end{array}$} & \multirow{2}{*}{$\begin{array}{c}\text { Linear Range } \\
\text { (ng/L) }\end{array}$} & \multirow{2}{*}{$\begin{array}{r}\text { LOD } \\
\text { (ng) }\end{array}$} & \multirow{2}{*}{$\begin{array}{r}\text { LOQ } \\
\text { (ng) }\end{array}$} & \multicolumn{2}{|c|}{ Precision (RSD, $n=6$ ) } & \multicolumn{2}{|c|}{ Repeatability $(n=6)$} & \multicolumn{2}{|c|}{ Recovery $(n=6)$} \\
\hline & & & & & Intra-Day (\%) & Inter-Day (\%) & Mean (mg/100g) & RSD (\%) & Mean (\%) & RSD (\%) \\
\hline A & $y=240.695+6301.38 x, 0.9998$ & $0.12 \sim 11.15$ & 0.04 & 0.12 & 2.71 & 3.99 & 12.29 & 0.77 & 99.1 & 2.33 \\
\hline $\mathrm{C}$ & $y=264.791+9060.44 x, 0.9997$ & $0.10 \sim 9.95$ & 0.01 & 0.03 & 2.68 & 2.95 & 3.48 & 1.35 & 97.2 & 3.01 \\
\hline G & $y=1363.37+47042.3 x, 0.9999$ & $0.10 \sim 9.87$ & 0.01 & 0.03 & 2.85 & 3.32 & 11.68 & 0.83 & 98.5 & 2.70 \\
\hline I & $y=5409.88+74348.5 x, 0.9998$ & $0.12 \sim 11.68$ & 0.01 & 0.03 & 2.51 & 3.62 & 1.54 & 2.27 & 98.9 & 2.67 \\
\hline $\mathrm{T}$ & $y=166.893+2164.58 x, 0.9992$ & $0.10 \sim 9.92$ & 0.03 & 0.09 & 1.38 & 2.60 & 0.20 & 3.01 & 96.3 & 4.38 \\
\hline $\mathrm{U}$ & $y=-591.107+18137.6 x, 0.9993$ & $0.09 \sim 8.91$ & 0.03 & 0.09 & 2.93 & 3.33 & 7.30 & 1.01 & 98.6 & 2.39 \\
\hline$x$ & $y=3103.68+57804.6 x, 0.9998$ & $0.10 \sim 9.83$ & 0.01 & 0.03 & 1.82 & 2.04 & 0.12 & 2.68 & 100.06 & 4.88 \\
\hline $\mathrm{dA}$ & $y=295.259+5284.18 x, 0.9997$ & $0.12 \sim 11.50$ & 0.04 & 0.12 & 1.50 & 2.99 & 0.99 & 3.32 & 98.5 & 3.29 \\
\hline $\mathrm{dC}$ & $y=157.122+4241.14 x, 0.9971$ & $0.09 \sim 8.80$ & 0.03 & 0.09 & 3.70 & 4.29 & 0.23 & 3.40 & 96.9 & 3.98 \\
\hline dG & $y=1235.86+44695.6 x, 1.0000$ & $0.09 \sim 8.77$ & 0.01 & 0.03 & 2.87 & 3.28 & 0.42 & 2.88 & 97.7 & 3.74 \\
\hline $\mathrm{dU}$ & $\mathrm{y}=-13.5999+1756.23 \mathrm{x}, 0.9988$ & $0.09 \sim 9.16$ & 0.03 & 0.09 & 2.48 & 3.58 & 0.37 & 3.95 & 100.12 & 4.05 \\
\hline AMP & $y=440.578+8906.34 x, 0.9989$ & $0.10 \sim 10.40$ & 0.01 & 0.03 & 3.25 & 3.97 & 0.75 & 3.45 & 97.7 & 4.46 \\
\hline $\mathrm{CMP}$ & $y=-264.393+5715.24 x, 0.9998$ & $0.10 \sim 9.88$ & 0.01 & 0.03 & 3.56 & 4.42 & 2.74 & 1.65 & 99.6 & 3.59 \\
\hline GMP & $y=467.242+6476.87 x, 0.9978$ & $0.11 \sim 11.28$ & 0.01 & 0.03 & 3.46 & 4.49 & 3.80 & 1.83 & 98.4 & 3.03 \\
\hline UMP & $\mathrm{y}=-47.7751+7174.29 \mathrm{x}, 0.9996$ & $0.12 \sim 11.48$ & 0.01 & 0.03 & 2.64 & 2.92 & 0.41 & 2.36 & 95.8 & 4.20 \\
\hline
\end{tabular}




\subsection{Quality Evaluation of T. matsutake Samples}

Eighty batches of T. matsutake were collected by CDC staff from Sichuan province. The contents of 15 nucleic acid compounds were determined by the proposed UPLC-QqQ/MS method. The results are shown in Table 3.

The nucleic acid compounds in this study could be divided into three categories: nucleosides, deoxynucleosides, and mononuclear nucleotides. In general, the total content of nucleosides was the highest, followed by mononuclear nucleotides, and the content of deoxynucleosides was the lowest. Four nucleosides $(\mathrm{T}, \mathrm{X}, \mathrm{dU}$, and $\mathrm{dC}$ ) could not be detected in some samples. The mononuclear nucleotides are typical flavoring nucleotides and can significantly enhance the umami taste together with umami amino acid (aspartic acid and/or glutamic acid) [18].

The total content of nucleic acid compounds was $37.57 \pm 7.95 \mathrm{mg} / 100 \mathrm{~g}(\mu \pm \sigma$, average value \pm standard deviation), which suggests that there was little difference in nucleic acid content among samples from different regions. The data of the total content of nucleic acid compounds in 80 samples were further treated with the SPSS 19.0 statistical package. A descriptive statistical analysis was used according to the type of the data. To test significance, the t-test and variance analysis were used. The data of the total content of nucleic acid compounds conformed to a normal distribution since the results of the test of normality were $p=0.200(>0.05)$, skewness $=0.463(<1)$, and kurtosis $=0.23$ $(<1)$. The stem-and-leaf plot is shown in Figure 3. The reference range of the total content of nucleic acid compounds could be defined by normal distribution method. The low limit of the total content was calculated as $24.49 \mathrm{mg} / 100 \mathrm{~g}(\mu-1.645 \sigma$, at the 0.05 level, one-tailed). Among the results of the 80 samples used in this work, the actual distribution (exceeding $24.49 \mathrm{mg} / 100 \mathrm{~g}$ ) was $97.5 \%$, which was greater than $95 \%$ of the theoretical distribution. In conclusion, we suggest that the total content of nucleic acid compounds in T. matsutake should be higher than $24.49 \mathrm{mg} / 100 \mathrm{~g}$ to be a qualified product.

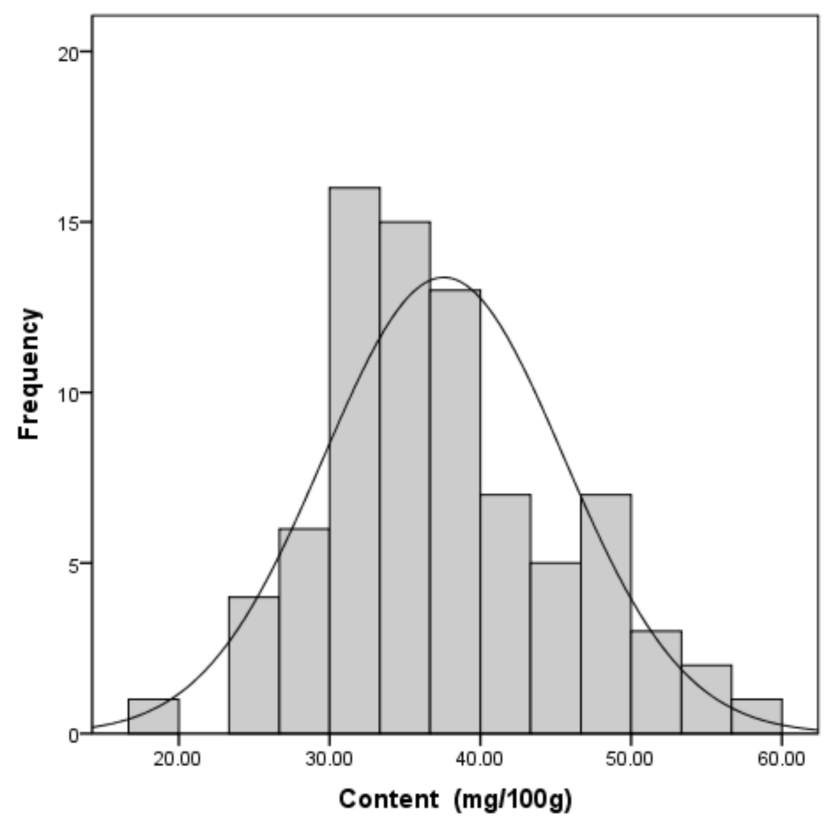

Figure 3. The stem-and-leaf plot of normal distribution plot. 
Table 3. The contents of 15 nucleic acid constituents in 80 T. matsutake samples (mg/100 g).

\begin{tabular}{|c|c|c|c|c|c|c|c|c|c|c|c|c|c|c|c|c|c|}
\hline No. & Location & $\mathbf{A}$ & $\mathrm{C}$ & G & I & $T$ & $\mathrm{U}$ & $x$ & $\mathrm{dA}$ & $\mathrm{dC}$ & $\mathrm{dG}$ & $\mathrm{dU}$ & AMP & CMP & GMP & UMP & Total \\
\hline S-01 & Jiulong & 8.41 & 2.49 & 6.97 & 1.07 & 0.01 & 5.09 & & 0.78 & 0.21 & 0.29 & & 0.87 & 2.32 & 3.06 & 0.52 & $32.08 \pm 2.73$ \\
\hline S-02 & Jiulong & 8.11 & 2.45 & 7.41 & 1.16 & 0.24 & 5.47 & & 0.60 & 0.14 & 0.32 & 0.06 & 0.88 & 2.31 & 3.24 & 0.47 & $32.87 \pm 2.75$ \\
\hline S-03 & Jiulong & 12.83 & 3.40 & 10.90 & 1.24 & 0.15 & 7.56 & & 0.69 & & 0.34 & & 0.88 & 3.16 & 4.69 & 0.53 & $46.39 \pm 4.35$ \\
\hline S-04 & Jiulong & 15.22 & 3.56 & 11.32 & 1.26 & 0.03 & 7.61 & & 0.86 & & 0.38 & 0.24 & 0.86 & 3.22 & 4.62 & 0.50 & $49.67 \pm 4.79$ \\
\hline S-05 & Jiulong & 12.29 & 3.48 & 11.68 & 1.54 & 0.20 & 7.30 & 0.12 & 0.99 & 0.23 & 0.42 & 0.37 & 0.75 & 2.74 & 3.80 & 0.41 & $46.33 \pm 4.11$ \\
\hline S-06 & Jiulong & 9.11 & 2.71 & 7.92 & 0.91 & & 5.56 & & 0.58 & 0.14 & 0.32 & 0.29 & 0.67 & 1.15 & 1.61 & 0.29 & $31.25 \pm 3.0$ \\
\hline S-07 & Jiulong & 7.89 & 1.93 & 6.10 & 0.94 & & 4.70 & & 0.37 & 0.02 & 0.19 & & 0.95 & 2.31 & 2.74 & 0.58 & $28.71 \pm 2.55$ \\
\hline S-08 & Jiulong & 14.53 & 3.28 & 11.02 & 1.38 & & 7.67 & & 0.61 & 0.05 & 0.32 & 0.33 & 0.87 & 2.54 & 3.87 & 0.40 & $46.87 \pm 4.64$ \\
\hline S-09 & Jiulong & 14.19 & 3.03 & 11.56 & 1.27 & & 7.29 & & 0.75 & 0.09 & 0.39 & 0.26 & 0.90 & 3.94 & 5.24 & 0.53 & $49.43 \pm 4.62$ \\
\hline S-10 & Jiulong & 10.44 & 2.97 & 8.17 & 1.15 & & 6.09 & & 0.87 & 0.29 & 0.43 & 0.22 & 1.07 & 2.92 & 4.06 & 0.54 & $39.22 \pm 3.32$ \\
\hline S-11 & Jiulong & 12.00 & 2.83 & 9.38 & 1.06 & 0.49 & 6.66 & & 0.92 & 0.08 & 0.36 & 0.20 & 0.46 & 1.69 & 2.96 & 0.39 & $39.46 \pm 3.80$ \\
\hline S-12 & Jiulong & 13.09 & 2.88 & 10.00 & 1.23 & & 6.69 & & 0.71 & 0.05 & 0.31 & 0.11 & 1.97 & 4.85 & 6.62 & 0.58 & $49.09 \pm 4.21$ \\
\hline S-13 & Jiulong & 7.84 & 1.90 & 6.61 & 0.86 & & 4.35 & & 0.47 & 0.00 & 0.24 & 0.10 & 1.62 & 2.88 & 3.24 & 0.53 & $30.65 \pm 2.55$ \\
\hline S-14 & Jiulong & 13.66 & 3.12 & 10.77 & 1.13 & 0.37 & 5.83 & & 0.77 & & 0.28 & 0.10 & 1.32 & 3.82 & 5.94 & 0.51 & $47.62 \pm 4.34$ \\
\hline S-15 & Jiulong & 14.70 & 2.50 & 10.99 & 1.11 & & 6.88 & & 1.01 & 0.17 & 0.34 & & 1.72 & 5.67 & 8.16 & 0.72 & $53.99 \pm 4.79$ \\
\hline S-16 & Jiulong & 10.64 & 2.73 & 9.31 & 1.22 & 0.67 & 5.92 & & 0.94 & & 0.49 & 0.15 & 0.82 & 2.43 & 3.41 & 0.45 & $39.17 \pm 3.4 \mathrm{c}$ \\
\hline S-17 & Jiulong & 14.93 & 3.32 & 12.35 & 1.58 & 0.16 & 8.24 & 0.03 & 0.91 & 0.17 & 0.41 & 0.15 & 1.12 & 3.66 & 4.88 & 0.48 & $52.38 \pm 4.73$ \\
\hline S-18 & Jiulong & 12.40 & 2.57 & 9.52 & 1.11 & 0.37 & 6.24 & & 0.77 & & 0.43 & & 0.75 & 2.42 & 3.35 & 0.47 & $40.40 \pm 3.98$ \\
\hline S-19 & Jiulong & 10.72 & 2.58 & 9.85 & 0.85 & & 6.93 & & 0.80 & & 0.40 & 0.19 & 1.20 & 2.87 & 3.53 & 0.41 & $40.34 \pm 3.75$ \\
\hline S-20 & Jiulong & 5.58 & 1.09 & 3.56 & 0.48 & 0.03 & 3.12 & & 0.20 & & 0.09 & & 0.49 & 1.07 & 1.49 & 0.48 & $17.68 \pm 1.73$ \\
\hline S-21 & Jiulong & 10.71 & 2.33 & 9.31 & 1.15 & 0.37 & 6.04 & & 0.70 & & 0.30 & 0.10 & 1.04 & 2.81 & 4.42 & 0.55 & $39.82 \pm 3.56$ \\
\hline$S-22$ & Jiulong & 11.06 & 2.54 & 8.82 & 1.06 & & 6.13 & & 1.09 & 0.21 & 0.47 & & 0.94 & 2.78 & 3.29 & 0.48 & $38.87 \pm 3.57$ \\
\hline S-23 & Jiulong & 15.45 & 2.97 & 11.81 & 1.17 & 0.21 & 7.98 & 0.01 & 1.21 & & 0.38 & & 1.50 & 6.67 & 8.79 & 0.72 & $58.88 \pm 5.10$ \\
\hline S-24 & Jiulong & 11.68 & 2.81 & 9.08 & 1.04 & & 6.10 & & 0.95 & 0.10 & 0.33 & 0.17 & 1.18 & 3.95 & 5.94 & 0.62 & $43.96 \pm 3.77$ \\
\hline S-25 & Jiulong & 11.92 & 3.76 & 11.02 & 1.14 & 0.44 & 7.40 & & 0.89 & 0.21 & 0.42 & 0.05 & 0.23 & 1.39 & 2.06 & 0.36 & $41.30 \pm 4.11$ \\
\hline S-26 & Jiulong & 14.04 & 2.82 & 10.35 & 1.32 & & 7.14 & & 0.64 & & 0.33 & 0.30 & 1.64 & 5.45 & 8.18 & 0.70 & $52.92 \pm 4.5$ \\
\hline S-27 & Jiulong & 12.63 & 2.11 & 8.23 & 1.12 & & 5.49 & 0.19 & 0.84 & 0.08 & 0.32 & & 2.18 & 8.02 & 8.97 & 0.94 & $51.12 \pm 4.22$ \\
\hline S-28 & Jiulong & 14.93 & 3.37 & 11.71 & 1.37 & 0.40 & 7.87 & 0.00 & 1.18 & 0.18 & 0.48 & 0.29 & & 5.23 & 6.38 & 0.62 & $54.04 \pm 4.78$ \\
\hline $\mathrm{S}-29$ & Jiulong & 10.35 & 3.53 & 6.95 & 1.61 & 0.49 & 6.23 & 0.05 & 1.42 & 0.12 & 0.48 & 0.35 & 2.70 & 5.36 & 6.59 & 0.66 & $46.90 \pm 3.22$ \\
\hline S-30 & Jiulong & 8.55 & 2.87 & 6.22 & 1.49 & 0.19 & 7.48 & 0.06 & 0.82 & & 0.37 & 0.36 & 2.70 & 3.86 & 5.41 & 0.77 & $41.15 \pm 2.91$ \\
\hline S-31 & Xiaojin & 9.30 & 2.19 & 6.76 & 1.28 & & 7.64 & & 0.44 & 0.03 & 0.28 & & 0.71 & 1.74 & 2.82 & 0.26 & $33.44 \pm 3.24$ \\
\hline S-32 & Xiaojin & 9.55 & 2.24 & 7.52 & 1.62 & 0.13 & 8.27 & 0.00 & 0.47 & & 0.19 & 0.24 & 0.79 & 1.66 & 2.37 & 0.16 & $35.22 \pm 3.33$ \\
\hline S-33 & Xiaojin & 6.52 & 1.25 & 5.90 & 1.21 & & 5.52 & 0.04 & 0.62 & & 0.33 & & 0.75 & 1.48 & 2.47 & 0.28 & $26.37 \pm 2.38$ \\
\hline S-34 & Xiaojin & 9.40 & 2.27 & 7.71 & 1.82 & & 8.49 & 0.08 & 0.44 & 0.08 & 0.32 & & 0.74 & 1.68 & 2.44 & 0.21 & $35.69 \pm 3.42$ \\
\hline S-35 & Xiaojin & 10.06 & 2.20 & 8.31 & 2.21 & & 11.26 & 0.03 & 0.30 & 0.10 & 0.35 & 0.14 & 0.77 & 1.61 & 2.51 & 0.19 & $40.03 \pm 3.94$ \\
\hline S-36 & Xiaojin & 8.95 & 2.15 & 7.18 & 1.66 & 0.28 & 8.45 & 0.05 & 0.46 & 0.09 & 0.28 & & 0.64 & 0.96 & 1.71 & 0.15 & $33.01 \pm 3.25$ \\
\hline S-37 & Xiaojin & 9.20 & 2.04 & 7.22 & 1.84 & 0.38 & 10.02 & 0.06 & 0.44 & & 0.25 & 0.11 & 0.47 & 1.05 & 1.71 & 0.12 & $34.93 \pm 3.53$ \\
\hline S-38 & Xiaojin & 9.51 & 2.32 & 7.14 & 1.83 & 0.17 & 7.96 & 0.02 & 0.34 & & 0.21 & & 0.75 & 1.45 & 2.25 & 0.18 & $34.13 \pm 3.31$ \\
\hline S-39 & Xiaojin & 7.79 & 2.11 & 6.04 & 1.05 & 0.27 & 6.66 & & 0.41 & 0.10 & 0.24 & & & 0.65 & 0.84 & 0.13 & $26.30 \pm 2.88$ \\
\hline S-40 & Xiaojin & 7.48 & 1.73 & 5.71 & 1.07 & 0.23 & 6.41 & & 0.25 & 0.04 & 0.18 & 0.03 & 0.35 & 0.53 & 0.80 & 0.11 & $24.91 \pm 2.64$ \\
\hline S-41 & Xiaojin & 7.92 & 2.02 & 6.72 & 1.64 & 0.26 & 6.98 & & 0.22 & 0.06 & 0.20 & & 0.64 & 1.28 & 1.80 & 0.19 & $29.94 \pm 2.8$ \\
\hline S-42 & Xiaojin & 6.48 & 2.04 & 5.67 & 1.45 & 0.41 & 6.09 & & 0.50 & & 0.32 & 0.05 & & 0.68 & 0.89 & 0.14 & $24.72 \pm 2.4 \mathrm{C}$ \\
\hline
\end{tabular}


Table 3. Cont

\begin{tabular}{|c|c|c|c|c|c|c|c|c|c|c|c|c|c|c|c|c|c|}
\hline No. & Location & A & C & G & I & $T$ & $\mathrm{U}$ & $x$ & $\mathrm{dA}$ & $\mathrm{dC}$ & $\mathrm{dG}$ & $\mathrm{dU}$ & AMP & CMP & GMP & UMP & Total \\
\hline S-43 & Xiaojin & 10.13 & 2.14 & 8.16 & 1.76 & 0.25 & 9.41 & & 0.38 & 0.08 & 0.26 & & 0.77 & 1.58 & 2.44 & 0.16 & $37.52 \pm 3.72$ \\
\hline S-44 & Xiaojin & 9.54 & 1.87 & 7.14 & 1.49 & 0.24 & 8.51 & 0.12 & 0.50 & 0.10 & 0.33 & & 1.53 & 2.62 & 4.11 & 0.31 & $38.41 \pm 3.30$ \\
\hline S-45 & Xiaojin & 8.80 & 1.99 & 5.93 & 1.23 & & 7.44 & 0.08 & 0.33 & 0.02 & 0.17 & 0.07 & 0.40 & 1.06 & 1.71 & 0.18 & $29.41 \pm 2.99$ \\
\hline S-46 & Xiaojin & 8.99 & 2.05 & 7.43 & 1.49 & 0.39 & 8.33 & 0.01 & 0.47 & 0.13 & 0.21 & 0.14 & 0.96 & 1.83 & 2.60 & 0.22 & $35.26 \pm 3.17$ \\
\hline S-47 & Xiaojin & 8.71 & 2.22 & 6.82 & 1.46 & 0.59 & 7.12 & & 0.30 & & 0.21 & 0.08 & 0.59 & 1.28 & 2.04 & 0.20 & $31.61 \pm 3.03$ \\
\hline S- 48 & Xiaojin & 10.60 & 2.40 & 7.67 & 1.76 & 0.28 & 9.31 & 0.02 & 0.37 & 0.15 & 0.26 & 0.10 & 0.61 & 1.51 & 2.80 & 0.18 & $38.02 \pm 3.60$ \\
\hline S- 49 & Xiaojin & 9.17 & 1.83 & 7.13 & 2.11 & 0.46 & 8.24 & & 0.38 & 0.08 & 0.31 & 0.23 & 1.08 & 1.78 & 2.73 & 0.23 & $35.76 \pm 3.18$ \\
\hline S-50 & Xiaojin & 8.72 & 2.11 & 7.22 & 1.41 & 0.35 & 7.26 & & 0.64 & 0.09 & 0.45 & 0.29 & 0.57 & 1.53 & 2.24 & 0.21 & $33.10 \pm 3.01$ \\
\hline S-51 & Xiaojin & 7.93 & 1.79 & 6.37 & 1.31 & 0.34 & 7.78 & & 0.33 & 0.10 & 0.26 & 0.31 & 1.00 & 1.55 & 2.33 & 0.21 & $31.60 \pm 2.87$ \\
\hline S-52 & Xiaojin & 9.73 & 2.02 & 6.90 & 1.89 & 0.23 & 9.44 & 0.07 & 0.43 & 0.07 & 0.31 & 0.09 & 1.33 & 2.36 & 3.46 & 0.28 & $38.60 \pm 3.37$ \\
\hline S-53 & Xiaojin & 8.80 & 1.96 & 5.95 & 1.71 & 0.21 & 7.79 & 0.01 & 0.38 & 0.03 & 0.16 & 0.15 & 0.41 & 0.89 & 1.42 & 0.22 & $30.09 \pm 2.97$ \\
\hline S-54 & Xiaojin & 7.80 & 1.89 & 6.73 & 2.13 & 0.08 & 8.20 & 0.04 & 0.25 & 0.02 & 0.21 & 0.15 & 0.66 & 1.43 & 1.75 & 0.19 & $31.52 \pm 2.94$ \\
\hline S-55 & Xiaojin & 6.92 & 1.95 & 6.17 & 1.04 & 0.17 & 6.33 & & 0.49 & & 0.33 & 0.08 & 0.72 & 1.17 & 1.91 & 0.24 & $27.52 \pm 2.56$ \\
\hline S-56 & Xiaojin & 8.44 & 1.96 & 6.63 & 1.27 & 0.45 & 7.73 & 0.03 & 0.45 & 0.14 & 0.24 & 0.21 & 0.58 & 1.18 & 2.11 & 0.19 & $31.63 \pm 2.93$ \\
\hline S-57 & Xiaojin & 7.17 & 1.86 & 6.33 & 1.62 & 0.50 & 5.89 & & 0.26 & 0.11 & 0.21 & 0.26 & 0.72 & 1.10 & 1.53 & 0.19 & $27.74 \pm 2.51$ \\
\hline S-58 & Xiaojin & 7.94 & 1.62 & 6.10 & 1.47 & 0.15 & 8.19 & & 0.27 & & 0.18 & 0.07 & 0.66 & 1.32 & 1.64 & 0.16 & $29.75 \pm 3.01$ \\
\hline S-59 & Xiaojin & 10.36 & 2.36 & 8.51 & 2.06 & & 9.45 & 0.11 & 0.42 & 0.03 & 0.36 & 0.17 & 0.99 & 2.31 & 3.65 & 0.23 & $41.01 \pm 3.71$ \\
\hline S-60 & Lixian & 8.41 & 2.49 & 6.97 & 1.07 & 0.01 & 5.09 & & 0.78 & 0.21 & 0.29 & & 0.87 & 2.32 & 3.06 & 0.52 & $32.09 \pm 2.74$ \\
\hline S-61 & Lixian & 8.11 & 2.45 & 7.41 & 1.16 & 0.24 & 5.47 & & 0.6 & 0.14 & 0.32 & 0.06 & 0.88 & 2.31 & 3.24 & 0.47 & $32.86 \pm 2.75$ \\
\hline S-62 & Lixian & 8.24 & 2.16 & 6.87 & 1.09 & 0.15 & 6.87 & & 0.54 & 0.11 & 0.30 & 0.03 & 0.73 & 2.14 & 2.97 & 0.25 & $32.45 \pm 2.87$ \\
\hline S-63 & Lixian & 9.34 & 2.38 & 8.57 & 1.26 & 0.22 & 6.51 & 0.02 & 0.41 & 0.16 & 0.33 & 0.11 & 0.81 & 1.92 & 3.87 & 0.19 & $36.10 \pm 3.20$ \\
\hline S-64 & Lixian & 8.15 & 2.61 & 7.12 & 1.18 & 0.15 & 5.95 & 0.01 & 0.32 & 0.07 & 0.19 & 0.09 & 0.67 & 1.59 & 2.18 & 0.16 & $30.44 \pm 2.76$ \\
\hline S-65 & Lixian & 9.42 & 2.34 & 6.93 & 1.02 & 0.24 & 6.81 & 0.04 & 0.27 & 0.05 & 0.26 & 0.10 & 0.89 & 1.85 & 2.63 & 0.21 & $33.06 \pm 3.03$ \\
\hline S-66 & Muli & 12.83 & 3.4 & 10.9 & 1.24 & 0.15 & 7.56 & & 0.69 & & 0.34 & & 0.88 & 3.16 & 4.69 & 0.53 & $46.37 \pm 4.35$ \\
\hline S-67 & Muli & 15.22 & 3.56 & 11.32 & 1.26 & 0.03 & 7.61 & & 0.86 & & 0.38 & 0.24 & 0.86 & 3.22 & 4.62 & 0.5 & $49.68 \pm 4.79$ \\
\hline S-68 & Muli & 12.64 & 2.14 & 9.76 & 1.01 & 0.08 & 8.29 & 0.02 & 0.35 & 0.03 & 0.39 & 0.33 & 0.91 & 2.37 & 3.34 & 0.36 & $42.02 \pm 4.05$ \\
\hline S-69 & Muli & 13.95 & 2.17 & 8.79 & 1.76 & 0.17 & 9.73 & & 0.37 & & 0.31 & 0.05 & 0.72 & 2.51 & 5.31 & 0.18 & $46.02 \pm 4.53$ \\
\hline S-70 & Kangding & 9.3 & 2.19 & 6.76 & 1.28 & & 7.64 & & 0.44 & 0.03 & 0.28 & & 0.71 & 1.74 & 2.82 & 0.26 & $33.45 \pm 3.24$ \\
\hline S-71 & Kangding & 9.55 & 2.24 & 7.52 & 1.62 & 0.13 & 8.27 & & 0.47 & 0.06 & 0.27 & 0.24 & 0.79 & 1.66 & 2.37 & 0.16 & $35.35 \pm 3.33$ \\
\hline S-72 & Kangding & 10.34 & 1.99 & 6.93 & 1.37 & 0.3 & 9.78 & 0.07 & 0.54 & 0.15 & 0.33 & 0.18 & 0.93 & 1.46 & 3.57 & 0.23 & $38.17 \pm 3.54$ \\
\hline S-73 & Kangding & 7.99 & 2.07 & 8.25 & 1.82 & 0.24 & 8.73 & 0.02 & 0.49 & 0.11 & 0.24 & 0.36 & 0.85 & 2.87 & 2.28 & 0.19 & $36.51 \pm 3.18$ \\
\hline S-74 & Kangding & 8.34 & 1.68 & 7.61 & 1.46 & 0.26 & 8.26 & & 0.32 & & 0.37 & 0.27 & 0.67 & 1.98 & 2.72 & 0.19 & $34.13 \pm 3.20$ \\
\hline S-75 & Luding & 9.54 & 2.11 & 8.38 & 2.21 & 0.15 & 7.96 & & 0.44 & 0.09 & 0.21 & 0.15 & 0.82 & 2.74 & 1.78 & 0.85 & $37.43 \pm 3.35$ \\
\hline S-76 & Luding & 8.83 & 2.17 & 8.18 & 1.84 & 0.28 & 6.67 & 0.07 & 0.54 & 0.1 & 0.19 & 0.27 & 0.77 & 2.93 & 1.9 & 0.34 & $35.08 \pm 3.03$ \\
\hline S-77 & Luding & 8.14 & 2.53 & 9.34 & 1.49 & 0.07 & 8.29 & 0.06 & 0.47 & 0.14 & 0.31 & 0.33 & 0.59 & 2.35 & 2.34 & 0.56 & $37.01 \pm 3.29$ \\
\hline S-78 & Yajiang & 8.92 & 1.97 & 8.16 & 1.05 & 0.21 & 8.31 & & 0.39 & & 0.18 & 0.09 & 0.83 & 1.67 & 1.1 & 0.66 & $33.54 \pm 3.40$ \\
\hline S-79 & Yajiang & 9.37 & 2.28 & 7.38 & 1.83 & 0.02 & 9.27 & 0.03 & 0.5 & 0.08 & 0.3 & 0.29 & 0.91 & 1.92 & 3.65 & 0.48 & $38.31 \pm 3.35$ \\
\hline \multirow[t]{2}{*}{ S-80 } & Yajiang & 8.16 & 2.54 & 7.91 & 1.17 & 0.19 & 8.99 & 0.02 & 0.51 & 0.05 & 0.28 & 0.24 & 0.56 & 1.85 & 2.91 & 0.53 & $35.91 \pm 3.22$ \\
\hline & & $10.08 \pm 2.40$ & $2.40 \pm 0.55$ & $\begin{array}{c}8.11 \pm \\
1.84\end{array}$ & $\begin{array}{c}1.38 \pm \\
0.35\end{array}$ & $\begin{array}{c}0.25 \pm \\
0.15\end{array}$ & $\begin{array}{c}7.32 \pm \\
1.42\end{array}$ & $\begin{array}{c}0.05 \pm \\
0.04\end{array}$ & $\begin{array}{c}0.58 \pm \\
0.26\end{array}$ & $\begin{array}{c}0.10 \pm \\
0.06\end{array}$ & $\begin{array}{c}0.30 \pm \\
0.08\end{array}$ & $\begin{array}{c}0.18 \pm \\
0.10\end{array}$ & $\begin{array}{c}0.92 \pm \\
0.45\end{array}$ & $\begin{array}{c}2.39 \pm \\
1.38\end{array}$ & $3.34 \pm 1.80$ & $0.37 \pm 0.20$ & \\
\hline
\end{tabular}




\section{Material and Methods}

\subsection{Chemicals and Reagents}

Eighty dried samples of T. matsutake were collected in accordance with official sampling requirements [19] from the mountain areas of Xiaojin County, Jiulong County, Yajiang County, Kangding County, Muli County, and Lixian County in Sichuan Province, China. Fifteen authentic standards of adenosine (A), cytidine (C), guanosine (G), inosine (I), thymidine (T), uridine (U), xanthosine dehydrate $(\mathrm{X}), 2^{\prime}$-deoxyadenosine (dA), 2'-deoxycytidine (dC), 2'-deoxyguanosine $(\mathrm{dG})$, $2^{\prime}$-deoxyuridine $(\mathrm{dU})$, adenosine $5^{\prime}$-monophosphate (AMP), cytidine $5^{\prime}$-monophosphate (CMP), guanosine $5^{\prime}$-monophosphate (GMP), and uridine $5^{\prime}$-monophosphate (UMP) were obtained from Sigma-Aldrich (St. Louis, MO, USA). The Milli-Q water purification system was used to prepare ultra-pure water for the UPLC analysis (Millipore, Bedford, MA, USA). The solvent ammonium acetate, acetonitrile, and diethylamine with LC-MS grade for UPLC-MS analysis were also purchased from Sigma-Aldrich (Sigma-Aldrich, St.Louis, MO, USA). Other chemicals and solvents of analytical grade were purchased from Sinopharm Chemical Reagent Co., Ltd. (Shanghai, China).

\subsection{Preparation of Standard Solution}

Stock solutions of 15 target analytes were prepared by dissolving each $10 \mathrm{mg}$ authentic standard in $100 \mathrm{~mL}$ ultra-pure water. Then, $1 \mathrm{~mL}$ of each of the 15 stock solutions was transferred to a $100 \mathrm{~mL}$ volumetric flask and diluted with ultra-pure water to obtain the working solution I of mixed standards with a concentration of approximately $10 \mu \mathrm{g} / \mathrm{mL}$. Mixed working solutions II-V were obtained by diluting working solution I with respective concentrations of about $7.5 \mu \mathrm{g} / \mathrm{mL}, 5 \mu \mathrm{g} / \mathrm{mL}, 2.5 \mu \mathrm{g} / \mathrm{mL}$, and $0.1 \mu \mathrm{g} / \mathrm{mL}$. All solutions were stored at $4{ }^{\circ} \mathrm{C}$ before use.

\subsection{Sample Preparation}

One gram of T. matsutake powder was accurately weighed into a $100 \mathrm{~mL}$ volumetric flask, about $90 \mathrm{~mL}$ ultra-pure water was added, and this was kept in a boiling water bath for $15 \mathrm{~min}$. Then, this was cooled to room temperature, the volume was fixed by ultra-pure water, and the extract of the T. matsutake sample was obtained. Finally, the solutions were filtered through a $0.22 \mu \mathrm{m}$ syringe filter into HPLC vials for UPLC analysis.

\subsection{Scanning and Identification of Nucleic Acid Compounds by UPLC-TOF/MS}

The Shimadzu UPLC system (Shimadzu, Kyoto, Japan) consists of pumps (LC-30AD), an online degasser (DGU-20A ${ }^{5 R}$ ), an auto-sampler (SIL-30AC), and a column oven (CTO-30aHE). Chromatographic separation was performed on a Thermo Hypercarb analytical column $(2.1 \mathrm{~mm} \times 100 \mathrm{~mm}, 5 \mu \mathrm{m})$ at $40{ }^{\circ} \mathrm{C}$. The mobile phase consisted of an aqueous phase $(2 \mathrm{mmol} / \mathrm{L}$ ammonium acetate solution containing $0.06 \%$ diethylamine, $\mathrm{pH} 11$ ) and an organic phase (acetonitrile containing $2 \mathrm{mmol} / \mathrm{L}$ ammonium acetate and $0.06 \%$ diethylamine). The linear gradient elution was $2 \% \sim 15 \% \sim 40 \% \sim 60 \%$ organic phase at $0 \sim 4 \sim 7.5 \sim 10 \mathrm{~min}$. The constant flow rate was $0.6 \mathrm{~mL} / \mathrm{min}$. The sample solution of $2 \mu \mathrm{L}$ was injected into the UPLC system by the auto-sampler.

Time-of-flight mass spectrometry measurements were performed on a $4600 \mathrm{Q}$-TOF mass spectrometer (AB Sciex, Concord, CA, USA) equipped with an electrospray ionization source. The Q-TOF/MS analysis was performed in negative mode with the following parameters: Ion source gas 1 (GS1) $\left(\mathrm{N}_{2}\right)$ at 50 psi, ion source gas 1 (GS1) at 50 psi, curtain gas at 35 psi, a temperature of $500{ }^{\circ} \mathrm{C}$, and an ionspray voltage floating at $-4500 \mathrm{~V}$. The mass range was set to $m / z 100-1000$. The system was operated under Analyst 1.6 and Peak 2.0 (AB Sciex, Concord, CA, USA) and used an APCI negative calibration solution to calibrate the instrument's mass accuracy in real-time. 


\subsection{Determination of Nucleic Acid Compounds by UPLC-QqQ/MS}

Chromatographic separation was performed under the same conditions as those used in the UPLC-TOF/MS analysis described above. The QqQ/MS measurements were accomplished by a triple quadrupole mass spectrometer equipped with an electrospray ionization source (ThermoFischer, TSQ VANTAGE, Waltham, MA, USA). The MS spectra were acquired in negative ion mode. The quantitative analysis of the target analytes was performed under multi-reaction monitoring (MRM) mode. The parameters of the mass spectrometry detector (MSD) were as follows: A vaporizer temperature of $350^{\circ} \mathrm{C}$, a capillary temperature of $350^{\circ} \mathrm{C}$, an aux gas pressure of $10 \mathrm{Arb}$, a sheath gas pressure of $40 \mathrm{Arb}$, an ion sweep gas pressure of $2 \mathrm{Arb}$, a discharge current of $4.0 \mu \mathrm{A}$, and a spray voltage of $-2000 \mathrm{~V}$. Data collection and processing were conducted with Thermo Xcalibur Workstation (Version 2.2, ThermoFischer, TSQ VANTAGE, Waltham, MA, USA).

\section{Conclusions}

In the present study, the combined use of UPLC-TOF/MS and UPLC-QqQ/MS was applied for the identification and determination of nucleic acid compounds in T. matsutake, including adenosine, cytidine, guanosine, inosine, thymidine, uridine, xanthosine dehydrate, $2^{\prime}$-deoxyadenosine, $2^{\prime}$-deoxycytidine, $2^{\prime}$-deoxyguanosine, 2'-deoxyuridine, adenosine $5^{\prime}$-monophosphate, cytidine $5^{\prime}$-monophosphate, guanosine $5^{\prime}$-monophosphate, and uridine $5^{\prime}$-monophosphate. The content of each nucleic acid compound was determined by the proposed UPLC-MS method in 80 T. matsutake samples collected from different regions in Sichuan province, Southwest China. The results show, for the first time, that T. matsutake is rich in nucleic acid compounds, and there is little difference in the nucleic acid content of T. matsutake from different locations in the Sichuan region. This shows that nucleic acid compounds should be incorporated into the quality standards as markers of T. matsutake and its products in the future.

Supplementary Materials: The following are available online.

Author Contributions: Data curation, Y.X.; Funding acquisition, Y.X., L.-S.Q. and J.X.; Methodology, Y.X., X.-M.H. and J.X.; Project administration, J.X.; Resources, W.J., X.-S.X., L.Y. and B.H.; Supervision, L.-S.Q.; Writing - original draft, L.-S.Q.; Writing - review \& editing, J.X.

Funding: This work was supported by the Chinese Academy of Sciences (CAS) 'Light of West China' Program, Self-supporting Research Project of Sichuan Provincial Centers for Disease Control and Prevention (No. ZX201613), the Collaborative Innovation Center of Sichuan for Elderly Care and Health, Chengdu Medical College (No. YLZBZ1820).

Conflicts of Interest: The authors declare no conflict of interest.

\section{References}

1. Kim, S.S.; Lee, J.S.; Cho, J.Y.; Kim, Y.E.; Hong, E.K. Process development for mycelial growth and polysaccharide production in Tricholoma matsutake liquid culture. J. Biosci. Bioeng. 2010, 109, 351-355. [CrossRef] [PubMed]

2. Chen, Y.; Du, X.J.; Zhang, Y.; Liu, X.H.; Wang, X.D. Ultrasound extraction optimization, structural features, and antioxidant activity of polysaccharides from Tricholoma matsutake. J. Zhejiang Univ.-Sci. B 2017, 18, 674-684. [CrossRef] [PubMed]

3. Hoshi, H.; Iijima, H.; Ishihara, Y.; Yasuhara, T.; Matsunaga, K. Absorption and tissue distribution of an immunomodulatory $\alpha$-D-glucan after oral administration of Tricholoma matsutake. J. Agric. Food Chem. 2008, 56, 7715-7720. [CrossRef] [PubMed]

4. Li, Q.; Wang, Y.Z.; Chen, Y.L.; Teng, M.Y.; He, J.; Wang, X.H.; Kong, F.G.; Teng, L.R.; Wang, D. Investigation of the immunomodulatory activity of Tricholoma matsutake mycelium in cyclophosphamide-induced immunosuppressed mice. Mol. Med. Rep. 2017, 16, 4320-4326. [CrossRef] [PubMed]

5. Ikekawa, T.; Uehara, N.; Maeda, Y.; Nakanishi, M.; Fukuoka, F. Antitumor activity of aqueous extracts of edible mushrooms. Cancer Res. 1969, 29, 734-735. [PubMed] 
6. Yang, S.; Ren, X.; Sheng, J.; Lu, J.; Li, T.; Tang, F.; Wang, Y.; Meng, L.; Meng, Q.; Teng, L. Preparation and the antitumor activity in vitro of polysaccharides from Tricholoma matsutake. World J. Microb. Biotechnol. 2010, 26, 497-503. [CrossRef]

7. Ying, X.H.; Ma, J.F.; Liang, Q.L.; Wang, Y.M.; Bai, G.; Luo, G.A. Identification and analysis of the constituents in an aqueous extract of Tricholoma matsutake by HPLC coupled with diode array detection/electrospray ionization mass spectrometry. J. Food Sci. 2013, 78, C1173-C1182. [CrossRef] [PubMed]

8. Jacobson, K.A.; Costanzi, S.; Ohno, M.; Joshi, B.V.; Besada, P.; Xu, B.; Tchilibon, S. Molecular recognition at purine and pyrimidine nucleotide (P2) receptors. Curr. Top. Med. Chem. 2004, 4, 805-819. [CrossRef] [PubMed]

9. Wachowius, F.; Attwater, J.; Holliger, P. Nucleic acids: Function and potential for abiogenesis. Q. Rev. Biophys. 2017, 50, e4. [CrossRef] [PubMed]

10. Local Standards for the Safety of Tricholoma Matsutake and Its Products, DBS 51/006-2018, Health Commission of Sichuan Province \& Sichuan Food and Drug Administration. Available online: http: / / www.scwst.gov.cn/xx/gsgg/201807/t20180704_17504.html (accessed on 20 July 2018).

11. Chen, C.; Xue, Y.; Li, Q.-M.; Wu, Y.; Liang, J.; Qing, L.-S. Neutral loss scan—based strategy for integrated identification of amorfrutin derivatives, new peroxisome proliferator-activated receptor gamma agonists, from Amorpha Fruticosa by UPLC-QqQ-MS/MS and UPLC-Q-TOF-MS. J. Am. Soc. Mass Spectrom. 2018, 29, 685-693. [CrossRef] [PubMed]

12. Qing, L.-S.; Xue, Y.; Liu, Y.-M.; Liang, J.; Xie, J.; Liao, X. Rapid magnetic solid-phase extraction for the selective determination of isoflavones in soymilk using baicalin-functionalized magnetic nanoparticles. J. Agric. Food Chem. 2013, 61, 8072-8078. [CrossRef] [PubMed]

13. Geis-Asteggiante, L.; Nuñez, A.; Lehotay, S.J.; Lightfield, A.R. Structural characterization of product ions by electrospray ionization and quadrupole time-of-flight mass spectrometry to support regulatory analysis of veterinary drug residues in foods. Rapid Commun. Mass Spectrom. 2014, 28, 1061-1081. [CrossRef] [PubMed]

14. Chen, Y.J.; Liang, Z.T.; Zhu, Y.; Xie, G.Y.; Tian, M.; Zhao, Z.Z.; Qin, M.J. Tissue-specific metabolites profiling and quantitative analyses of flavonoids in the rhizome of Belamcanda chinensis by combining laser-microdissection with UHPLC-Q/TOF-MS and UHPLC-QqQ-MS. Talanta 2014, 130, 585-597. [CrossRef] [PubMed]

15. Gross, M.L. Accurate masses for structure confirmation. J. Am. Soc. Mass Sp. 1994, 5, 57. [CrossRef]

16. Sun, W.-X.; Zhang, Z.-F.; Xie, J.; He, Y.; Cheng, Y.; Ding, L.-S.; Luo, P.; Qing, L.-S. Determination of a astragaloside IV derivative LS-102 in plasma by ultra-performance liquid chromatography-tandem mass spectrometry in dog plasma and its application in a pharmacokinetic study. Phytomedicine 2019, 53, $243-251$. [CrossRef]

17. Xie, J.; Li, J.; Liang, J.; Luo, P.; Qing, L.-S.; Ding, L.-S. Determination of contents of catechins in oolong teas by quantitative analysis of multi-components via a single marker (QAMS) method. Food Anal. Methods 2017, 10, 363-368. [CrossRef]

18. Kinnamon, S.C. Umami taste transduction mechanisms. Am. J. Clin. Nutr. 2009, 90, S753-S755. [CrossRef] [PubMed]

19. Tricholoma matsutake, GB/T 23188-2008, National Standard of the People's Republic of China. Available online: http:/ / www.gb688.cn/bzgk/gb/newGbInfo?hcno=EE887FA3F6717085FB88AFF35C8D092B (accessed on 1 June 2009).

Sample Availability: Samples of the all compounds are available from the authors. 